Check for updates

Cite this: RSC Adv., 2018, 8, 28613

Received 8th June 2018

Accepted 31st July 2018

DOI: $10.1039 / \mathrm{c} 8 \mathrm{ra04904a}$

rsc.li/rsc-advances

\title{
Degradation characterization and pathway analysis of chlortetracycline and oxytetracycline in a microbial fuel cell
}

\author{
Ji Wang, ${ }^{\text {abcd }}$ Boyi Zhou, ${ }^{\text {bcd }}$ Ruijia Ge, ${ }^{\text {e }}$ Tian-shun Song, ${ }^{\text {bcd }}$ Jinping Yu ${ }^{\star a}$ \\ and Jingjing Xie (D) *bcd
}

\begin{abstract}
The wide presence of antibiotics in the environment has raised concerns about their potential impact on ecological and human health. This study was conducted to evaluate the degradation of antibiotics (chlortetracycline (CTC) and oxytetracycline (OTC)) in microbial fuel cells (MFCs) and the change of toxicity. The degradation rates of $60 \mathrm{mg} \mathrm{L}^{-1}$ CTC and OTC in the MFCs were $74.2 \%$ and $78 \%$, respectively, within 7 days. The degradation ability of the two antibiotics followed the order of OTC > CTC. Toxicity test results of the zebrafish illustrated the toxicity of OTC and CTC was largely eliminated by MFC treatment. Furthermore, possible degradation pathways of CTC and OTC were speculated using LC-MS analysis. High-throughput sequencing analysis indicated that Petrimonas, Azospirillum, Dokdonella, Burkholderia and Stenotrophomonas were the predominant genera in the MFC anode biofilm. Therefore, this work is of great significance for future studies on the treatment of antibiotics in wastewater by MFCs.
\end{abstract}

\section{Introduction}

The development of human society is facing many challenges, especially environmental issues, which deserve particular attention. Water, solid-waste, and antibiotic pollution restricts our sustainable development. ${ }^{1}$ Chinese antibiotics production had reached 162000 tons in 2013, and more than 50000 tons of antibiotics had been discharged into water and soil environment. ${ }^{2}$ Tetracyclines, such as tetracycline (TC), chlortetracycline (CTC), and oxytetracycline (OTC), are the most commonly used antibiotics. TCs are often used in the breeding of livestock and poultry because of their low cost and high efficiency. ${ }^{3,4}$ However, these antibiotics can harm people, animals, and plants in varying degrees. ${ }^{5,6}$ TCs could not be completely absorbed by a living organism, and the absorption rate is only approximately $30 \%{ }^{7,8} \mathrm{TCs}$ have a stable structure and can easily form insoluble refractory complex. ${ }^{9}$

Several common treatment methods of TCs are advanced oxidation, activated carbon adsorption, and microbial degradation. ${ }^{10-12}$ The mechanism of chemical oxidation of TCs was the

${ }^{a}$ Institute of Botany, Jiangsu Province and Chinese Academy of Sciences, Nanjing 210014, PR China. E-mail: 664841805@qq.com

${ }^{b}$ State Key Laboratory of Materials-Oriented Chemical Engineering, Nanjing Tech University, Nanjing 211816, PR China. E-mail: xiej@njtech.edu.cn

${ }^{c}$ College of Life Science and Pharmaceutical Engineering, Nanjing Tech University, Nanjing 211816, PR China

${ }^{d}$ Jiangsu National Synergetic Innovation Center for Advanced Materials (SICAM), Nanjing 211816, PR China

${ }^{e}$ The Madeira School, Virginia 22102, USA reaction of ozone with hydroxides in water, a process which releases free radicals to degrade TCs. Andreozzi et al. ${ }^{13}$ used $11 \mathrm{mg} \mathrm{L}{ }^{-1}$ of ozone to degrade OTC, which was completely degraded after $20 \mathrm{~min}$. However, chemical oxidation presents evident disadvantages, such as high energy consumption, nonselective oxidation of free radicals, and production of toxic by-products. ${ }^{14}$ Meanwhile, activated carbon adsorption removes contaminants from the aqueous environment using the many adsorption sites in porous materials. However, antibiotics are difficult to remove completely using this process. In 2009, Nolwenn et al. ${ }^{15}$ studied the degradation of CTC and OTC using microorganisms. They found that microorganisms did not degrade CTC and OTC very well, but the antibiotics were well adsorbed by the microorganisms. Moreover, high concentrations of antibiotics killed the microorganisms. Consequently, the microbial removal rate of TCs was low. Therefore, a new efficient and inexpensive method is urgently needed to degrade TCs.

The microbial fuel cell (MFC) is a new organic pollutant treatment by combining microorganisms with electrochemical systems. ${ }^{16}$ The MFC can convert energy and produce electricity

Table 1 Concentrations of the glucose-oxytetracycline (OTC) mixtures and glucose-chlortetracycline (CTC) mixtures in the microbial fuel cell (MFC) during acclimation stage

\begin{tabular}{lrrrrrrrr}
\hline Acclimation stage & A & B & C & D & E & F & G & H \\
\hline Glucose $\left(\mathrm{mg} \mathrm{L}^{-1}\right)$ & 1000 & 1000 & 1000 & 1000 & 1000 & 500 & 0 & 0 \\
OTC $\left(\mathrm{mg} \mathrm{L}^{-1}\right)$ & 10 & 20 & 30 & 40 & 50 & 60 & 60 & 60 \\
CTC $\left(\mathrm{mg} \mathrm{L}^{-1}\right)$ & 10 & 20 & 30 & 40 & 50 & 60 & 60 & 60
\end{tabular}


Table 2 Gradient elution program for the analysis of OTC and CTC

Elution time

\begin{tabular}{lrrrrrrrr}
$(\min )$ & 0 & 2 & 2.1 & 7 & 10 & 11 & 14 & 16 \\
\hline A (\%) & 92 & 82 & 75 & 78 & 65 & 65 & 60 & 92 \\
B (\%) & 0 & 0 & 5 & 4 & 10 & 15 & 15 & 0 \\
C (\%) & 8 & 18 & 20 & 18 & 25 & 20 & 25 & 8
\end{tabular}

Table 3 Gradient elution program for the analysis of OTC and CTC degradation products in LC-MS

\begin{tabular}{lrr}
\hline Time $(\min )$ & A\% & B\% \\
\hline 0.0 & 95 & 5 \\
5.0 & 95 & 5 \\
6.0 & 72 & 28 \\
17.0 & 72 & 28 \\
18.0 & 65 & 35 \\
28.0 & 65 & 35 \\
30.0 & 95 & 5 \\
31.0 & 95 & 5
\end{tabular}

while degrading organic matter, ${ }^{17,18}$ such as easily degradable $^{19,20}$ and bio-refractory organics. ${ }^{21,22}$ Several studies have recently focused on the degradation of TCs by $\mathrm{MFC}^{23,24}$ to improve TC removal. However, the characterization and degradation mechanisms of the different kinds of TCs by MFC remain unknown.

In this study, we inoculated anaerobic microorganisms in the anode of an MFC. Then, the degradation rates of CTC and OTC were studied, and the toxicity of the degradation products after MFC treatment was analyzed. A possible degradation pathway of CTC and OTC was proposed. Finally, the microbial community activity was tested to reveal the relationship between antibiotic degradation and microbial activity.

\section{Materials and methods}

\section{MFC construction and operation}

The dual-chamber MFC reactor separated by a proton exchange membrane (Nafion117, Dupont Co., USA) consisted of plexiglas material (net volume of $250 \mathrm{~mL}$ each). The anode and cathode were made of graphite felt $[50 \mathrm{~mm} \times 50 \mathrm{~mm} \times 5 \mathrm{~mm}$ (length $\times$ width $\times$ thickness)]. The anolyte consisted of glucose medium containing $1 \mathrm{~g} \mathrm{~L}^{-1}$ glucose, $11.53 \mathrm{~g} \mathrm{~L}^{-1} \mathrm{Na}_{2} \mathrm{HPO}_{4} \cdot 12 \mathrm{H}_{2} \mathrm{O}, 2.77 \mathrm{~g}$ $\mathrm{L}^{-1} \mathrm{NaH}_{2} \mathrm{PO}_{4} \cdot 2 \mathrm{H}_{2} \mathrm{O}, 0.31 \mathrm{~g} \mathrm{~L}^{-1} \mathrm{NH}_{4} \mathrm{Cl}$, and $0.13 \mathrm{~g} \mathrm{~L}^{-1} \mathrm{KCl}$. The catholyte consisted of $2.77 \mathrm{~g} \mathrm{~L}^{-1} \mathrm{NaH}_{2} \mathrm{PO}_{4} \cdot 2 \mathrm{H}_{2} \mathrm{O}, 0.13 \mathrm{~g} \mathrm{~L}^{-1}$ $\mathrm{KCl}, 11.53 \mathrm{~g} \mathrm{~L}^{-1} \mathrm{Na}_{2} \mathrm{HPO}_{4} \cdot 12 \mathrm{H}_{2} \mathrm{O}$, and $13.2 \mathrm{~g} \mathrm{~L}^{-1} \mathrm{~K}_{3} \mathrm{Fe}(\mathrm{CN})_{6}$. The anolyte and catholyte were added to the anode and cathode chambers of the MFC, respectively, while the external load of $1000 \Omega$ was fixed between the cathode and the anode. The temperature was maintained at $25{ }^{\circ} \mathrm{C}$. The MFC experiments
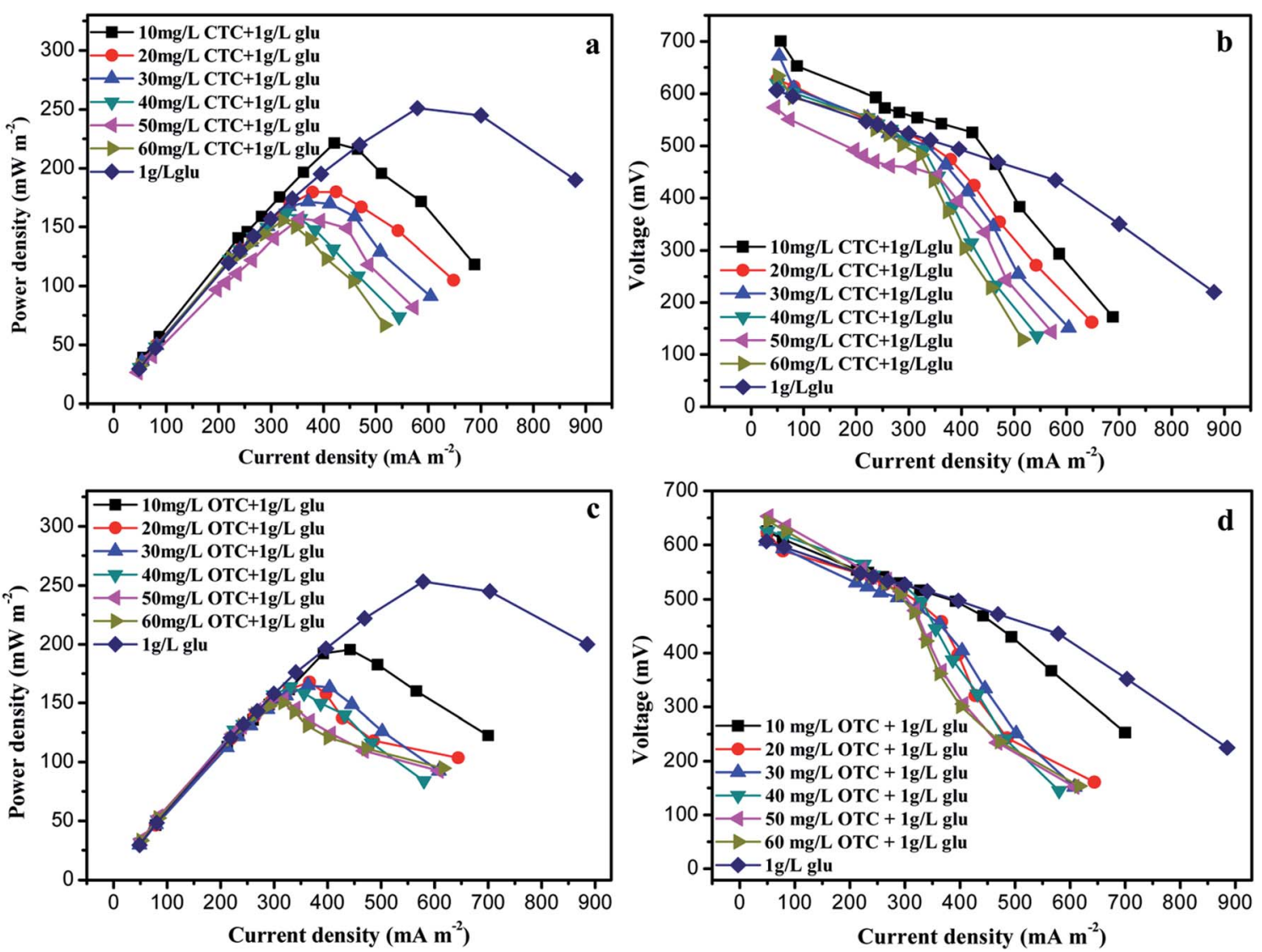

Fig. 1 Power density ( $a$ and $c$ ) and polarization curve (b and d) in the microbial fuel cell (MFC) with different concentrations of chlortetracycline (CTC) or oxytetracycline (OTC). 
were performed in duplicate under each experimental condition.

The experiments were divided into three stages, namely, startup, gradient acclimation, and removal stages. During the startup stage, $245 \mathrm{~mL}$ of the anolyte and $5 \mathrm{~mL}$ of the anaerobic activated sludge were added to the anode chamber of the MFC. The anolyte was changed every 7 days for a total of four weeks, and the voltage of the MFC was stabilized. During the gradient acclimation stage, without any anaerobic activated sludge, the anode chamber consisted of $250 \mathrm{~mL}$ of the anolyte and different concentrations of OTC and CTC (Table 1). According to the relevant literature ${ }^{25,26}$ on other antibiotic removal as well as our preliminary experiments, the $60 \mathrm{mg} \mathrm{L}^{-1}$ OTC or CTC without any glucose was used as sole substrate on the removal stage to evaluate the biodegradation effect of OTC or CTC in the MFC. The MFC reactors were operated in three groups, namely, closed circuit (CC), open circuit (OC), and nondomestication cycle (NC). The NC group did not undergo the gradient acclimation stage. The main difference between the CC and OC modes was whether a fixed external resistor was connected.

\section{Analyses}

The concentrations of OTC and CTC were detected by highperformance liquid chromatography (Agilent 1260, USA) with UV detection at $275 \mathrm{~nm}$. The OTC and CTC were separated by an Agilent Eclipse Plus C18 column $(4.6 \times 100 \mathrm{~mm}, 3.5 \mu \mathrm{m})$ at a flow rate was $1.0 \mathrm{~mL} \mathrm{~min}^{-1}$. The column was kept at $25^{\circ} \mathrm{C}$. The measurements of the flow phase gradient, which contained with $0.03 \%$ oxalic acid solution (A), methanol solution (B), and acetonitrile solution (C), are shown in Table 2. The OTC and CTC degradation products were analyzed by liquid chromatography-mass spectrometry (LC-MS; Agilent 1260, USA) and electrospray ionization-quadrupole time-of-flight MS. The source temperature was $350{ }^{\circ} \mathrm{C}$, and the ion spray voltage was $3500 \mathrm{~V}$. The nebulizer was set to $40 \mathrm{psig}$, and the flow of dry gas was set to $10.0 \mathrm{~L} \mathrm{~min}^{-1}$. The samples were analyzed using Eclipse XDB-C18 $(4.6 \times 150 \mathrm{~mm}, 5 \mu \mathrm{m})$. The mobile phase consisted of A and B. Phase A represented $0.1 \%$ formic acid solution, while phase B consisted of $40 \%$ methanol and $60 \%$ acetonitrile. The flow rate was controlled at $1.0 \mathrm{~mL} \mathrm{~min}^{-1}$, and the volume of injection was $30 \mu \mathrm{L}$. The flow phase gradients used to obtain the measurements are shown in Table 3.

A precision multimeter with a data acquisition system (Keithley 2700, USA) was used to collect the voltage at $10 \mathrm{~min}$ intervals. The external resistor was changed from $5000 \Omega$ to 100 $\Omega$ when the voltage output of the MFCs was steady. The current density and power density were calculated according to the projected anodic surface area. Current $(I)$ was calculated according to Ohm's law: $U=I R$, where $U$ is the voltage, and $R$ is the external resistance. Power $(P)$ was calculated as $P=I U$.

\section{Toxicity evaluation using the zebrafish}

The toxicity of the effluent of the MFCs was evaluated using the body length, hatching rate, survival rate, and heart rate of the zebrafish. Wild-type zebrafish (Tubingen line) were obtained from the Model Animal Research Center of Nanjing University.
The zebrafish were kept in a dedicated fish tank with the temperature maintained at $28.5{ }^{\circ} \mathrm{C}$, according to Li's report. ${ }^{27}$ The embryos were obtained by mating the male and female zebrafish in tanks, and the light-dark photoperiod was 14 $\mathrm{h}: 10 \mathrm{~h}$. The embryos were washed by an embryo medium $(0.2 \mathrm{~g}$ $\mathrm{L}^{-1}$ of Instant Ocean ${ }^{\circledR}$ salt in distilled water), while the malformed embryos were discarded. The authors declare that all procedures performed in studies involving live subjects were in accordance with the Ethical Standards of the Institutional and National Research Committee and with the 1964 Helsinki declaration and its later amendments or comparable ethical standards. Authors also state that all the zebrafish studies performed were approved by the Institutional Animal Care and Use Committee (IAUC) of Nanjing Tech University.

The embryos were randomly placed in a 48 -well plate at a density of 10 embryos, and each well contained $500 \mu \mathrm{L}$ of medium. Zebrafish embryos were treated with $60 \mathrm{mg} \mathrm{L}^{-1}$ of OTC and CTC, CC, OC, and NC after the MFC process. The control group did not have any OTC and CTC, while the other groups were treated with different concentrations of OTC or CTC or degradation products of OTC or CTC. The dead embryos were discarded during the whole experiment until $72 \mathrm{hpf}$ to reduce the impact of these embryos on the experiment. The body length was measured from the anterior-most portion of the head to the tip of the tail along the body axis. Heart rates (per 60
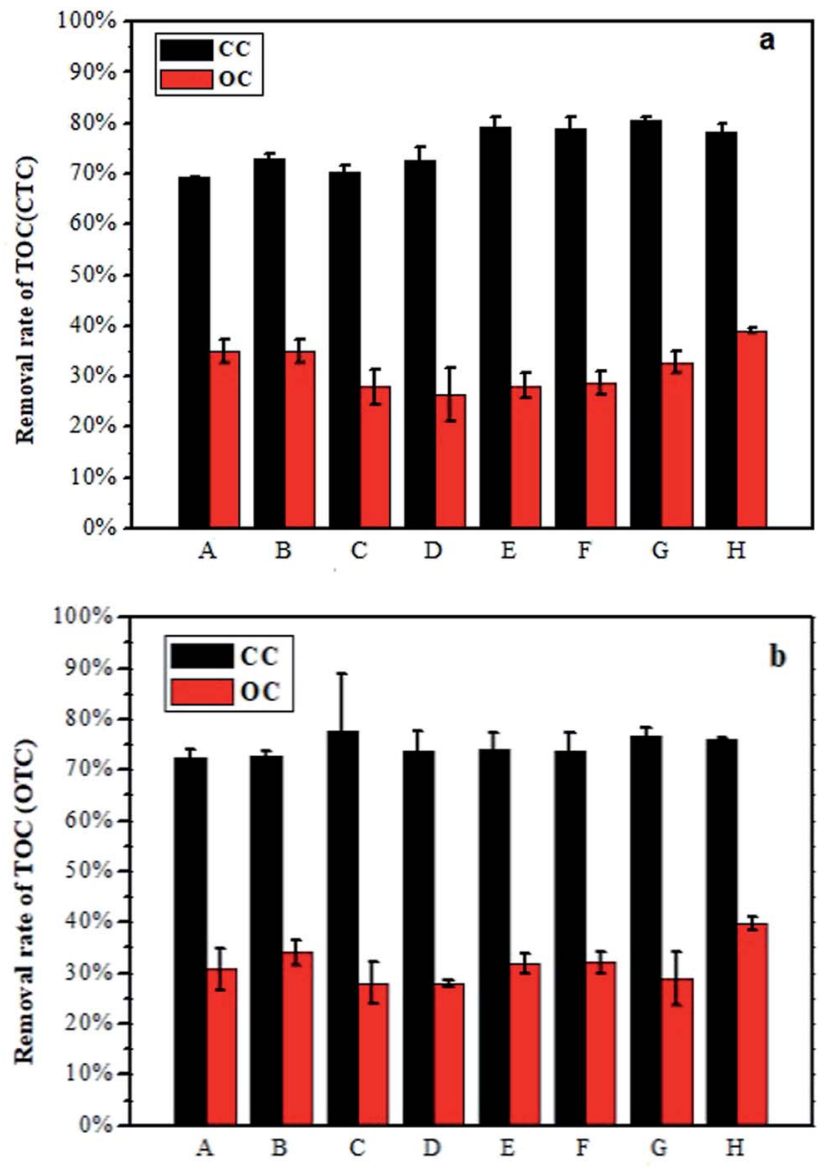

Fig. 2 Removal rate of total organic carbon in the MFC with CTC (a) or OTC (b). 

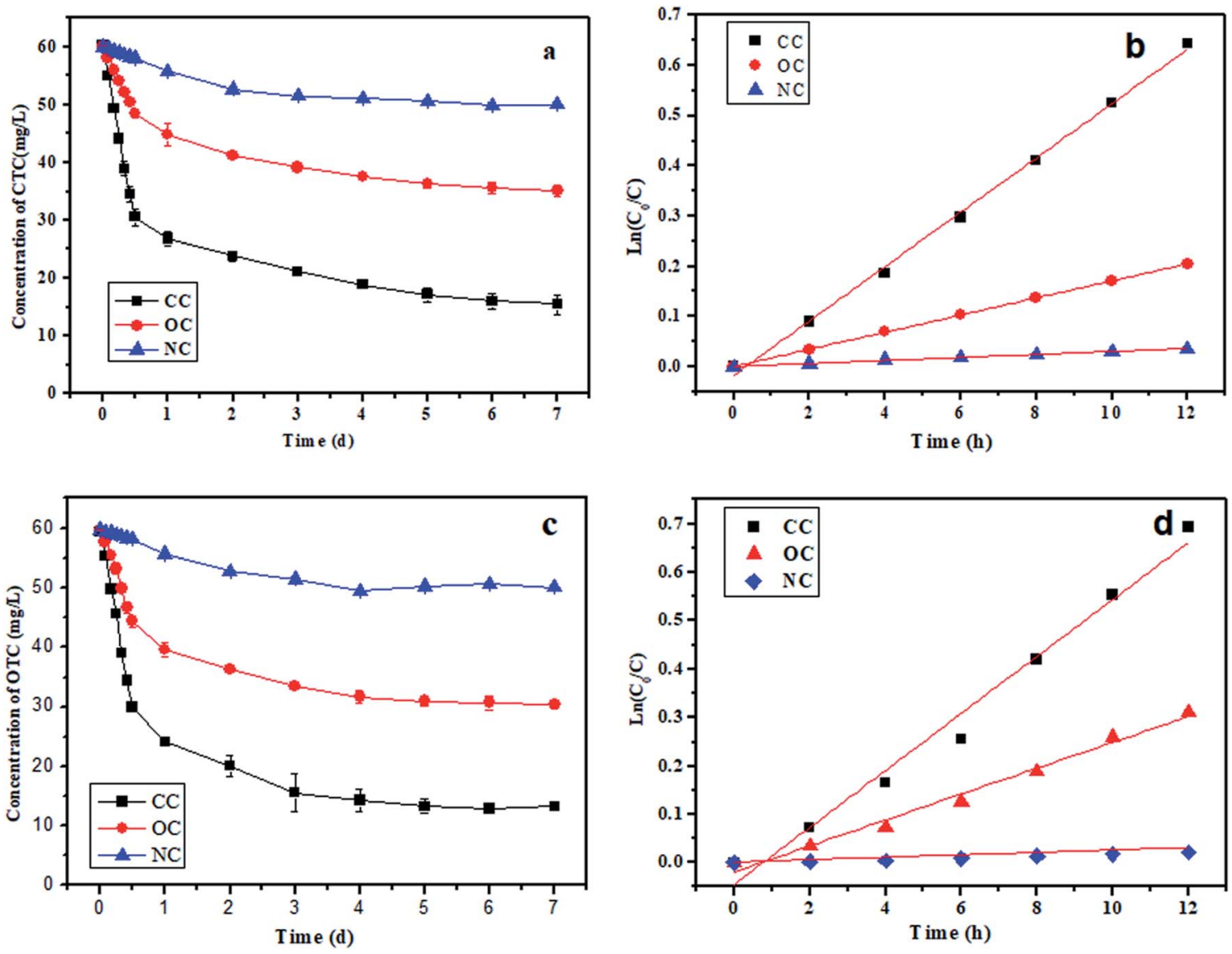

Fig. 3 (a) Variation in the CTC concentration over time; (b) kinetics curve of CTC degradation in the closed circuit (CC), open circuit (OC), and nondomestication cycle (NC) groups. (c) Variation in the OTC concentration over time; (d) kinetics curve of OTC degradation in the CC, OC, and NC groups.

s) were monitored using a dissecting microscope (Nikon, SMZ745T). The hatching and survival rates were recorded under the dissecting microscope. Experiments for each group were performed in triplicates to improve the accuracy of the experiment.

\section{Microbial diversity analysis}

The microbial community composition of the anode biofilm in the MFC was analyzed at the end of the experiment using bacterial 16S rDNA sequence analysis on an Illumina platform. The anodic biofilm was obtained using a sterile razor blade to vigorously scrape the electrodes. The PowerSoil $®$ DNA Isolation Kit (MO BIO Laboratories Inc., Carlsbad, CA, USA) was used according to the manufacturer's instructions to extract the DNA of the samples. DNA quality was measured using a Nano Drop ND-2000 Spectrophotometer (NanoDrop Technologies Inc., Wilmington, USA), and only the highly pure genomic DNA $\left(A_{260} /\right.$ $A_{280}=1.8$ ) was used for Illumina high-throughput sequencing by GENE DENONO (Guangzhou, China). Richness and biodiversity indices were received using the Mothur software package. The operational taxonomic units were classified using the $97 \%$ identity to the $16 \mathrm{~S}$ rRNA gene sequence as a cutoff. The reports of taxonomic classification were based on the sequences from the Ribosomal Database Project, from the phylum to the genus, with a bootstrap cutoff of $50 \%$. Finally, the microbial community map was created by the sequencing results.

Table 4 The $k$ values of the biodegradation kinetics of OTC and CTC

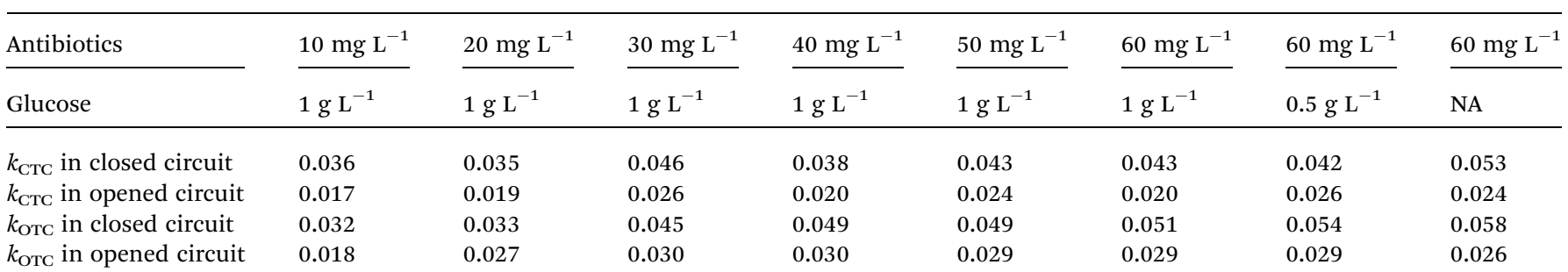




\section{Results and discussion}

\section{Electrochemical characteristics}

The electrochemical characteristics of the MFC were evaluated by measuring the power density when the MFC ran stably at the gradient acclimation stage. The maximum power density $\left(P_{\max }\right)$ of MFC without CTC or OTC was $252 \mathrm{~mW} \mathrm{~m}^{-2}$ (Fig. 1). When $10 \mathrm{mg} \mathrm{L}^{-1}$ CTC was added to the MFCs, the $P_{\max }$ reached $221.3 \mathrm{~mW} \mathrm{~m}^{-2}$ (Fig. 1a). $P_{\max }$ was significantly reduced during the gradient acclimation stage with increasing CTC concentration. Finally, the $P_{\max }$ of MFC with $1 \mathrm{~g}$ of glucose and $60 \mathrm{mg} \mathrm{L}{ }^{-1}$ CTC only reached $155.5 \mathrm{~mW} \mathrm{~m}^{-2}$. The trend of internal resistance was estimated from the polarization curves (Fig. 1b). When the CTC content increased in the MFCs, the internal resistance of the MFCs evidently increased from 305.6 $\Omega$ to $413.3 \Omega$, and $P_{\max }$ was reduced relatively. The same trend was observed in the MFC with OTC. The highest $P_{\max }(195.36$

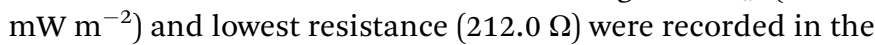
MFC with $10 \mathrm{mg} \mathrm{L}^{-1}$ OTC, followed by the MFC with 20$50 \mathrm{mg} \mathrm{L}{ }^{-1}$ OTC. By contrast, the MFCs with $60 \mathrm{mg} \mathrm{L}^{-1}$ OTC demonstrated the lowest $P_{\max }\left(150.41 \mathrm{~mW} \mathrm{~m}^{-2}\right)$ and yielded higher internal resistance (383.6 $\Omega$ ) (Fig. 1c and d). The experimental results might be due to the fact that the higher concentration of CTC and OTC affected the activity of the biofilm in MFC and thus influenced the generation of electricity by the MFC. ${ }^{28}$

\section{Total organic carbon (TOC) removal}

TOC usually represents the total amount of carbon in the organic matter. TOC is an important index for assessing the degradation of organic matter in water. ${ }^{29}$ The TOC removal efficiency of CTC is shown in Fig. 2a. The TOC removal efficiency in the MFCs with CTC under the CC was basically above $69 \%$. Compared with the CC, the MFCs with CTC under the OC had lower TOC removal efficiency, with the highest value of not more than $40 \%$. The TOC removal efficiency of OTC with CC was over $70 \%$ (Fig. 2b). Similarly, the removal rate of TOC in the MFCs with OTC under OC was lower than in the MFC with CC, with the former having the highest removal efficiency of only $39.7 \%$. The total TOC removal efficiencies of the two antibiotics were similar under CC or OC.

\section{Degradation of OTC and CTC}

The highest removal efficiencies of CTC and OTC were observed in the CC group (Fig. 3a and c). Approximately 50.8\% of CTC and $49.9 \%$ of OTC were degraded by the CC group within 0.5 day. The final concentrations of CTC and OTC in the effluent were $15.5 \mathrm{mg} \mathrm{L}^{-1}$ and $13.2 \mathrm{mg} \mathrm{L}^{-1}$, respectively, and the corresponding removal efficiencies reached up to $74.2 \%$ and $78 \%$ within 7 days. In Fig. 3a, only $19.2 \%$ and $3.4 \%$ removal rates were observed in the OC and NC groups within 0.5 day, respectively, while the final removal efficiencies of CTC in the OC and NA groups were $41.6 \%$
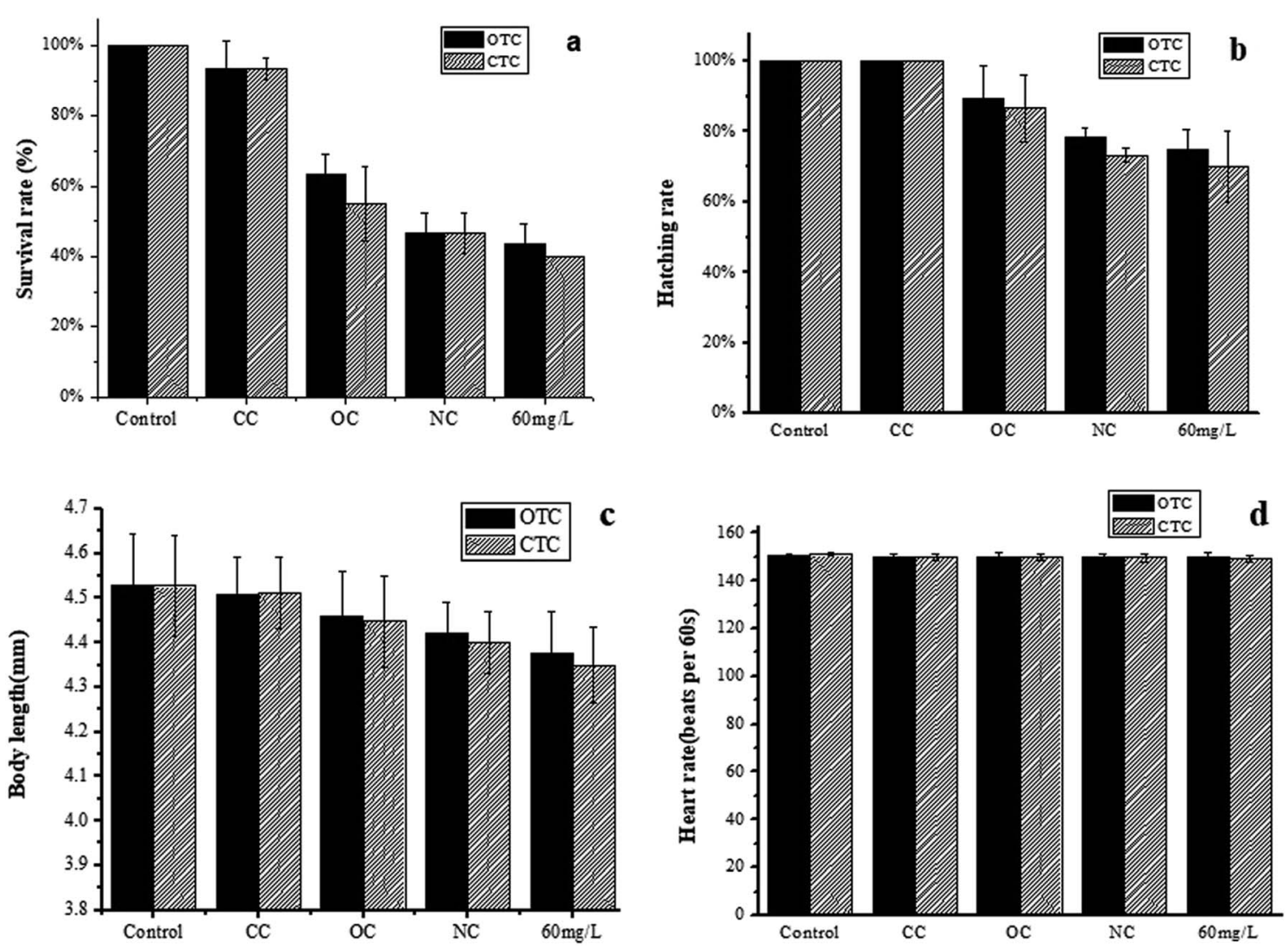

Fig. 4 Toxicity assessment of the different treatment groups. (a) Survival rate, (b) hatching rate, (C) body length, and (d) heart rate and of zebrafish. 
and $16.6 \%$, respectively, within 7 days. Similarly, the removal rates of OTC within 0.5 days and 7 days were lower in the OC and NC groups than in the CC group. This phenomenon illustrated the importance of the electron transfer and the gradient acclimation during the antibiotic removal by MFC.

A degradation kinetics model was established within 0.5 days to further determine the antibiotic degradation capability among the different groups. Substrate biodegradation was described with the following first-order kinetic model:

$$
\ln \left(C_{0} / C\right)=k \times t
$$

where $C$ is the concentration $\left(\mathrm{mg} \mathrm{L}^{-1}\right)$ of CTC or OTC during the experiment; $C_{0}$ is the initial concentration $\left(\mathrm{mg} \mathrm{L}^{-1}\right)$ of CTC or OTC; $k$ is the biodegradation kinetic constant $\left(\mathrm{h}^{-1}\right)$; and $t$ is time (h). The results of the linear curve fit of degradation over time are shown in Fig. $3 \mathrm{~b}$ and $\mathrm{d}$. The corresponding $k$ value was calculated. Table 4 shows that the $k$ value increased slowly in the CC groups containing glucose with increasing antibiotic concentration. After removing the glucose, the $k$ value did not decrease but increased, and the phenomenon also proved that the microorganisms could adapt to this system in the CC groups even in the absence of glucose as the carbon source. Meanwhile, the $k$ values of the CC groups were higher than of the OC group at any stage, while those of OTC were better than of CTC in the final stage of the CC or OC reactor. Hence, the removal rate of OTC was superior to CTC, probably because the structural formula of CTC contained chlorine atoms, and this characteristic might have affected the degradation activity of the microorganisms. ${ }^{30-32}$

The final stage of the CTC and OTC resulted in the highest $k$ values of $0.052 \mathrm{~h}^{-1}$ and $0.058 \mathrm{~h}^{-1}$, followed by $k_{\text {OC,СтC }}(0.024$ $\left.\mathrm{h}^{-1}\right), k_{\mathrm{OC}, \mathrm{OTC}}\left(0.026 \mathrm{~h}^{-1}\right), k_{\mathrm{NC}, \mathrm{CTC}}\left(0.0029 \mathrm{~h}^{-1}\right)$, and $k_{\mathrm{NC}, \mathrm{OTC}}$ $\left(0.0022 \mathrm{~h}^{-1}\right)$. The CTC degradation rate in the CC group was 18.3 times of that in the NC group, while the OTC degradation rate in the CC group was 26.4 times of that in the NC group.

The NC group represented common biological treatment and was studied as control group in our experiment, the degradation rate was very slow. The OC group represented anodic biofilm effect in MFC without external circuit connection. Although the OC group was worse than the CC group, the degradation of antibiotics in the OC group was better than in the NC group. This phenomenon emphasized the importance of domestication microorganisms for the antibiotic removal in wastewater by biological treatment. The CC group represented bioelectrochemistry effect in
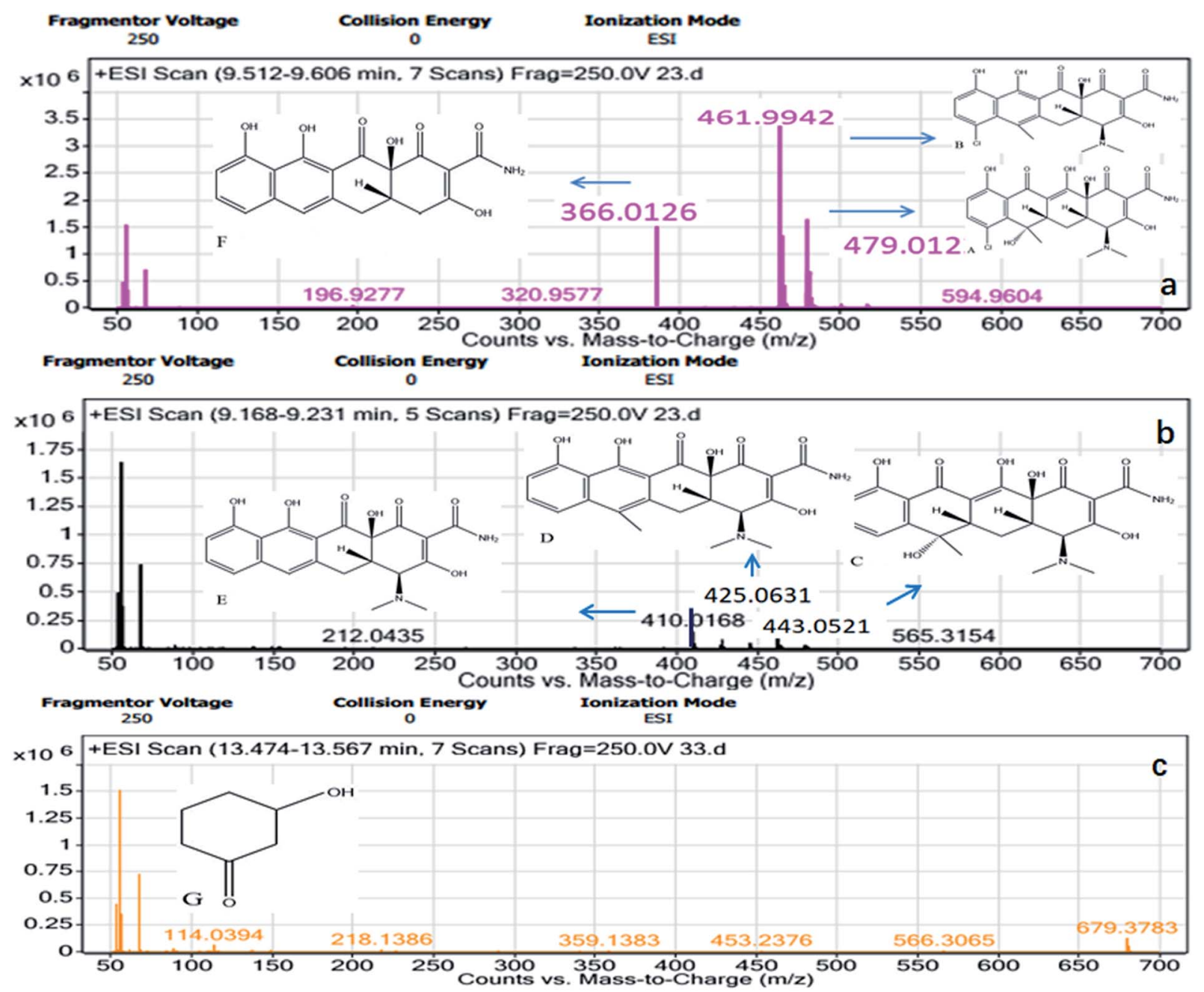

Fig. 5 Mass spectrometry (MS) identification of CTC degradation products. 
MFC and exhibited better degradation rate than the OC group, due to the generation of electrons caused by the oxidation of CTC and OTC on the anode. These electrons transferred to the terminal electron acceptor oxygen through the CC to complete the redox reaction. The electrode as a continuous long-term electron acceptor for anaerobic microorganism in the CC group, which enhanced the degradation of organic matter under anaerobic conditions. Therefore, the degradation of antibiotics was particularly faster under the CC. ${ }^{24}$

\section{Toxicity evaluation of zebrafish embryos}

The zebrafish exhibited high genetic homology to mammals and human. ${ }^{33,34}$ Thus, the zebrafish are often used to monitor the water environment, especially for toxicity assessment, developmental biology, and drug screening. ${ }^{35}$ Although the MFC with closed circuit could effectively degrade CTC and OTC, the toxicity of the degradation products needs to be evaluated. Therefore, the toxicity of the effluent after different treatments was evaluated by zebrafish.

The survival rate reflects the degree of harm of antibiotics to the zebrafish. Thus, this factor is a key indicator for evaluating the toxicity of the effluent. Only $43.3 \%$ of the zebrafish could survive in $60 \mathrm{mg} \mathrm{L}^{-1}$ OTC (Fig. 4a), and this value was higher than that in $60 \mathrm{mg} \mathrm{L}^{-1} \mathrm{CTC}(40 \%)$. However, the survival rates for embryos treated with OTC in the CC, OC, and NC group were $93.3 \%, 63.3 \%$, and $46.7 \%$, respectively. The survival rates of the CC, OC, and NC groups treated with CTC were 93.3\%, 55.0\%, and $46.7 \%$, respectively. Compared with the control (without OTC or CTC), the effluent in the CC group had weak effect on the survival rate of the zebrafish.

The hatching of the embryos apparently displayed different hydatoncus status in the different treatment groups among all toxicity tests. The hatching rate of the CC group treated by CTC or OTC was the same as the control (100\%; Fig. 4b). The hatching rates of the OC and NC groups in the OTC were $89.5 \%$ and $78.3 \%$, respectively, but these values were still higher than that of $60 \mathrm{mg} \mathrm{L}^{-1}$ OTC (75.0\%). Compared with the OTC, the hatching rate of CTC was lower. The OC, NC, and $60 \mathrm{mg} \mathrm{L}^{-1}$ of CTC were $86.6 \%, 73.3 \%$, and $70 \%$, respectively. The results showed that the effluent in the CC group had no effect on the hatching rate of the zebrafish. Meanwhile, the results showed that CTC were more toxic to zebrafish than OTC.
Fragmentor Voltage

250

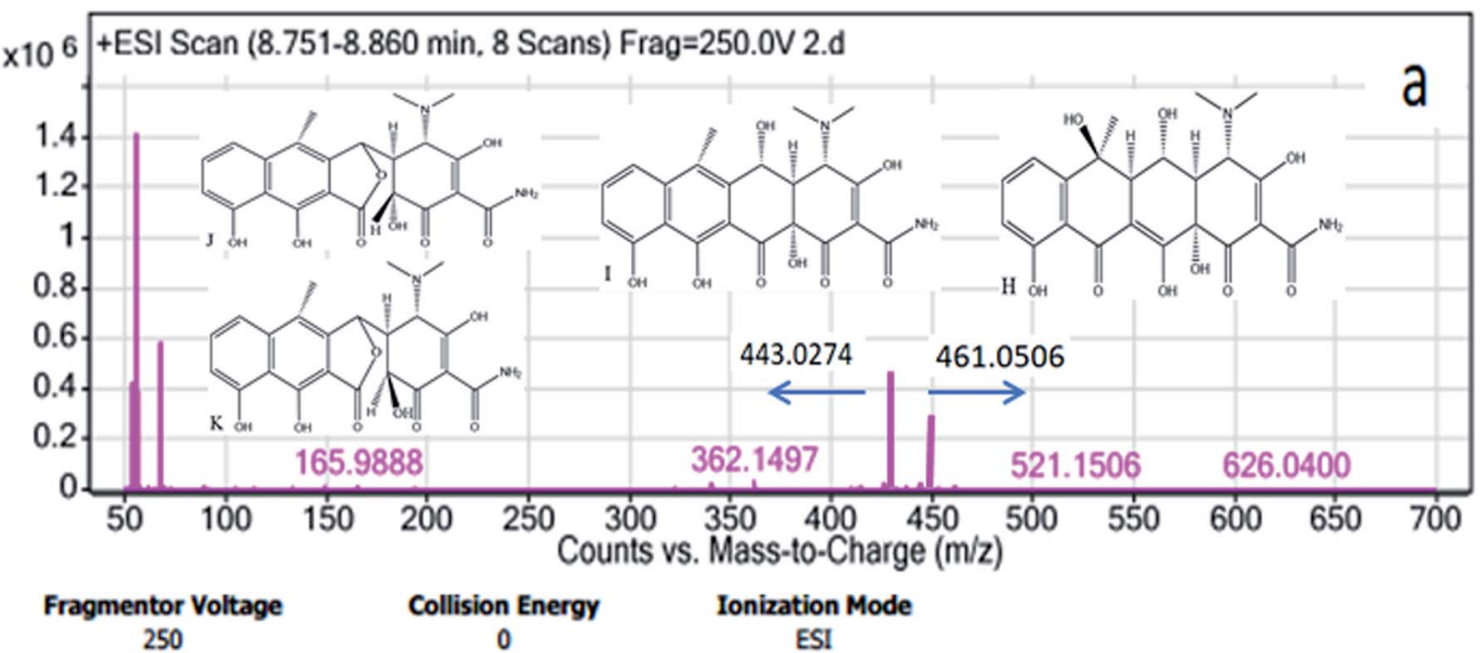

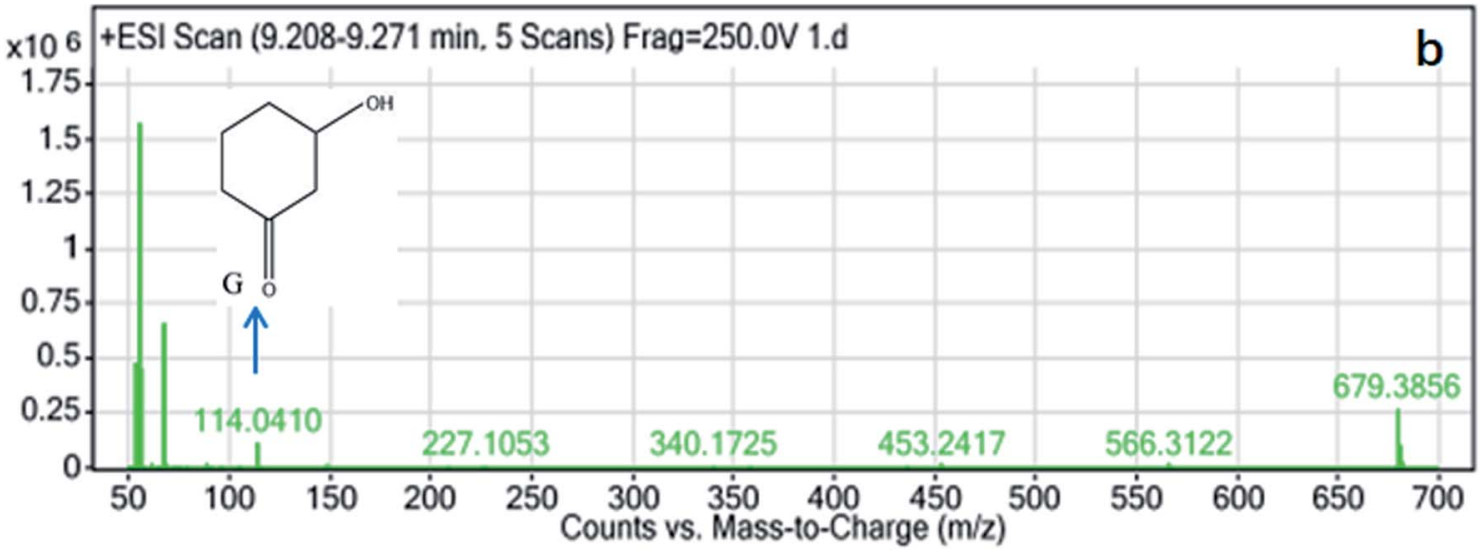

Fig. 6 MS identification of OTC degradation products. 
The body length (Fig. 4c) is an important indicator for the healthy growth of the zebrafish embryo. In the control group of the OTC, the body length of a healthy zebrafish embryo was $4.53 \mathrm{~mm}$ at $72 \mathrm{hpf}$, followed by the zebrafish in the CC (4.51 $\mathrm{mm}$ ), OC (4.46 mm), NC (4.42 mm), and $60 \mathrm{mg} \mathrm{L}^{-1}$ group (4.38 $\mathrm{mm}$ ) groups. Except for the CC group, other groups had remarkable or less impact to the body length of zebrafish embryo. The same phenomenon was observed in the CTC groups. The body lengths of the zebrafish embryos exposed to the control, CC, OC, NA, and $60 \mathrm{mg} \mathrm{L}^{-1}$ groups were 4.53, 4.51, $4.45,4.40$, and $4.35 \mathrm{~mm}$, respectively. The results indicated that the two antibiotics shortened the body length of zebrafish embryos, and CTC had a greater impact on body length of zebrafish embryos than OTC.

The heart rate of the zebrafish embryos reflects the degree of harm of the antibiotics to the cardiovascular system. The normal heartbeat of the control group was 150 beats per min, which was also the heart rates of the other groups. Thus, the OTC and CTC had no obvious effect on the heart rate at any of the test conditions.

Among all toxicity test parameters to the zebrafish embryos, the survival rate apparently displayed the most sensitivity in the experiments. The experimental results showed that the CC group had the lowest toxicity to zebrafish embryos than the other groups and was also closest to the control group. Both CTC and OTC had remarkable impact on the zebrafish embryos, and CTC was more toxic than OTC. However, after the MFC treatment with CC, OTC and CTC were remarkably degraded, and the degradation products showed nearly no toxicity.

\section{Possible degradation pathways of CTC and OTC}

To fully understand the degradation of CTC and OTC by MFC, the effluent samples were examined by LC-MS. The probable metabolites of CTC (Fig. 5) and OTC (Fig. 6) were identified by comparing their molar masses with previously reported compounds, such as dechlorinated chlortetracycline and dehydrated chlortetracycline. ${ }^{36-39}$

The possible degradation pathways of CTC (Fig. 7) and OTC (Fig. 8) were proposed according to the detected intermediate products at different sampling points. In the MFC, the degradation started directly from the bond breaking of a hydrogen atom and a hydroxyl group, yielding the compound dehydrated CTC (compound B), which was ionized with $\mathrm{m} / \mathrm{z} 461$. Subsequently, the compound of dechlorinated CTC (compound C) was the result of the loss of chlorine atom from CTC. Further oxidation of the dehydrated CTC and dechlorinated CTC led to compound D. After the oxidation of the lateral groups, the methyl of C6 position of compound D was broken to produce

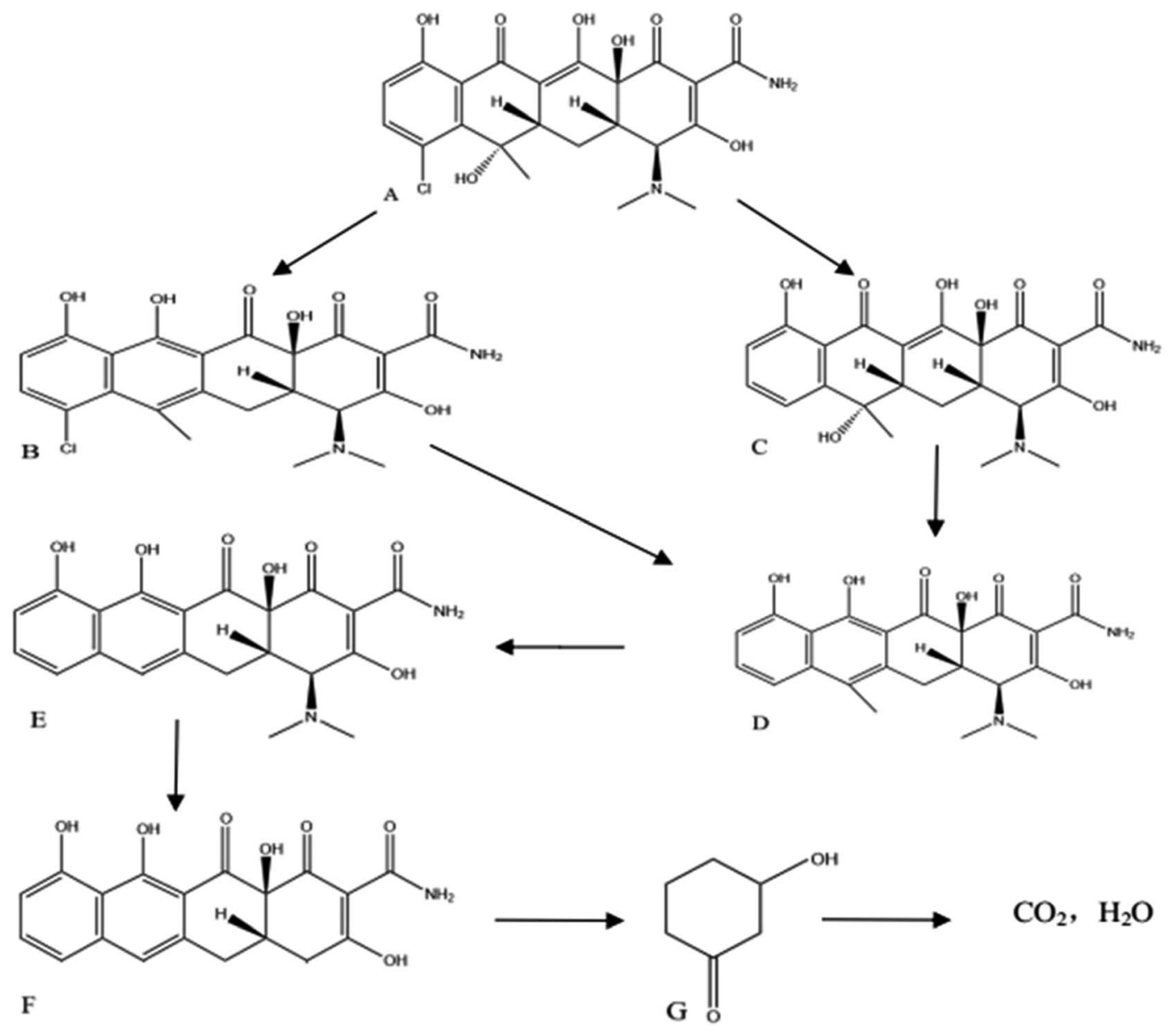

Fig. 7 Possible pathways for CTC degradation in the MFC. 
compound E, which was ionized with $\mathrm{m} / \mathrm{z}$ 410. In addition, product $\mathrm{F}(\mathrm{m} / \mathrm{z} 366)$ was also theoretically formed by loss of $-\mathrm{N}\left(\mathrm{CH}_{3}\right)_{2}$ group from the $\mathrm{C} 4$ position of compound E. Subsequently, the ring of the compound cracked and lost the $-\mathrm{CONH}_{2}$ group on the rightmost ring. Moreover, the double bond of the ring was further oxidized to form 3-hydroxycyclohexanone (compound G). Eventually, the 3-hydroxycyclohexanone was converted to $\mathrm{CO}_{2}$ and $\mathrm{H}_{2} \mathrm{O}$ with further hydroxyl radical attack. Similar behavior was observed during the degradation of chloramphenicol in bioelectrochemical systems. ${ }^{\mathbf{4 0}}$

The OTC degradation path was similar to the possible degradation pathways of CTC. In Fig. 8, the initial degradation started from the bond breaking of a hydrogen atom and a hydroxyl group of the carbon, forming anhydrooxytetracycline (AOTC, compound I). According to a previous study, under weak acidic conditions ( $\mathrm{pH} 3-6.5)$, the AOTC was quickly degraded to $\alpha$-APOTC (compound J) or/and $\beta$-APOTC (compound $\mathrm{K}) .{ }^{39}$ The $\mathrm{pH}$ in the experiment was between 6.5 and 7. Therefore, further oxidation of the C12 position of AOTC led to the production $\alpha$-APOTC or/and $\beta$-APOTC. However, AOTC, $\alpha$ APOTC, and $\beta$-APOTC were impossible to distinguish only by LC-MS, because the three compounds had the same $\mathrm{m} / \mathrm{z}$ values. These compounds were further oxidized and allowed to undergo ring-opening reactions to form 3-hydroxycyclohexanone (compound $\mathrm{G}$ ). Then, further hydroxyl radicals attack resulted in the formation of $\mathrm{CO}_{2}$ and $\mathrm{H}_{2} \mathrm{O}$. Therefore, the degradation products showed nearly no toxicity in the zebrafish toxicity test.

\section{Bacterial community composition}

The microbial communities of the biofilm in MFC reactors were analyzed at the end of the experiment. Proteobacteria was the most abundant phylum in all MFCs, followed by Firmicutes and Bacteroidetes (Fig. 9a). The relative abundance of Bacteroidetes in the CC of OTC $(10.6 \%)$ was higher than those of the OC of OTC (3.0\%). Similarly, Bacteroidetes $(10.1 \%)$ in the CC of CTC was more than the Bacteroidetes (7.0\%) in the OC of CTC.<smiles>C[C@H]1[C@@]2(C)C(=C(O)[C@@]3(O)C(=O)C(C(N)=O)=C(O)[C@H](N(C)C)[C@@]13C)C(=O)c1c(O)cccc1[C@@]2(C)O</smiles>

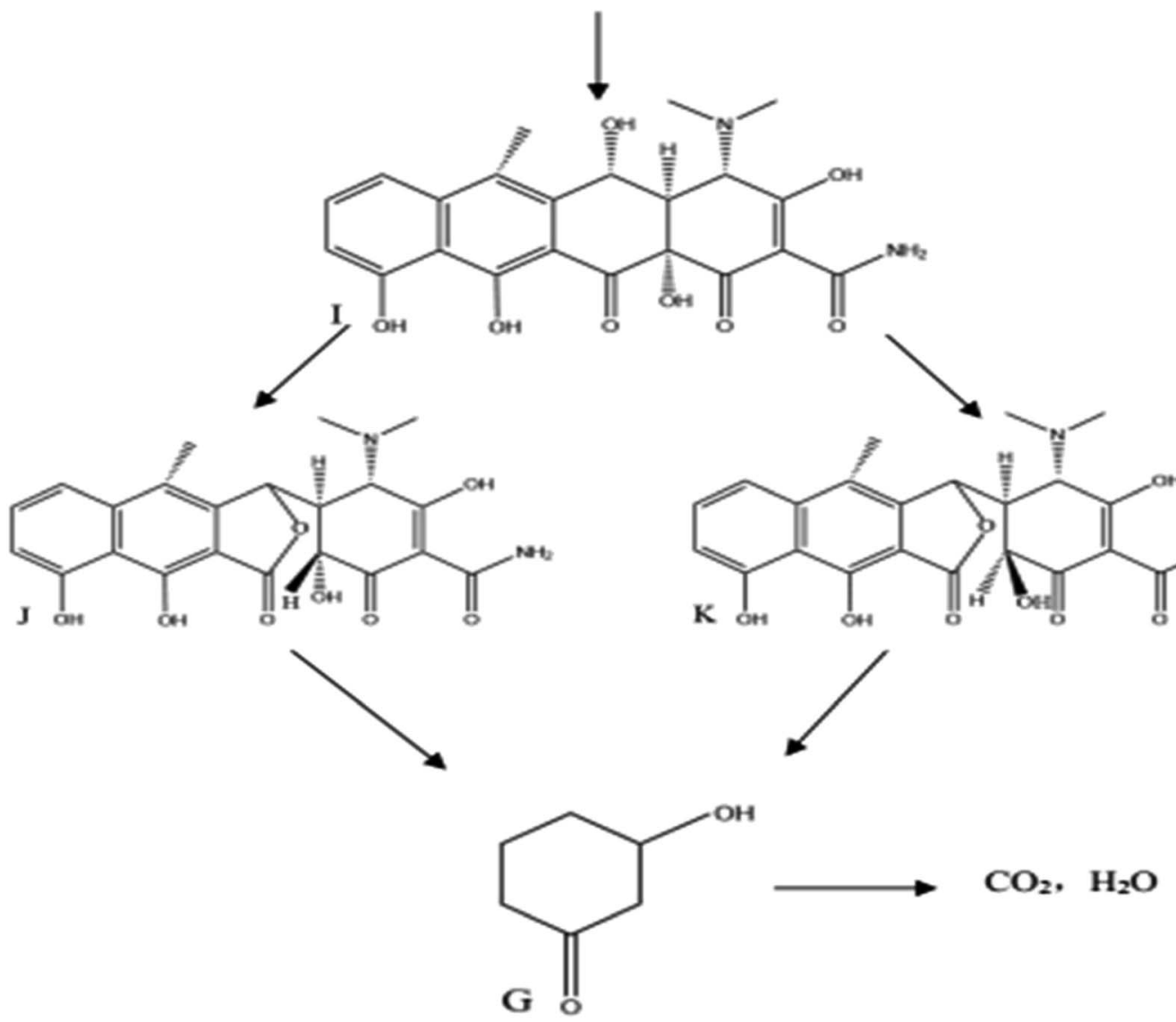

Fig. 8 Possible pathways for OTC degradation in the MFC. 
Stenotrophomonas (4.6\%), Azospirillum (4.1\%), and Pandoraea $(4.1 \%)$ were the most abundant genera in the CC of OTC, while Stenotrophomonas (17.2\%), Burkholderia (11.9\%), and Pandoraea (2.1\%) dominated in the OC of OTC (Fig. 9b). Stenotrophomonas was an acid-producing bacterium that can ferment carbohydrates to produce organic acid, ${ }^{\mathbf{4 1}}$ while Burkholderia was reported to use organic carbon to produce electron donor ${ }^{42}$ and could denitrify and produce nitrogen gas to maintain the anaerobic environment in an anode region. The abundance of Azospirillum in the CC of OTC was more than that in the OC of OTC. Azospirillum uses a wide range of carbon sources, such as glucose, fructose, and ethanol, which had been reported to be resistant to antibiotics ${ }^{\mathbf{4 3}}$ and fixed nitrogen. ${ }^{\mathbf{4 4}}$

In addition, the CC of CTC was dominated by Petrimonas (12.0\%), Azospirillum (6.4\%), and Dokdonella (4.2\%) in the relative abundance of genera, while the OC of CTC mainly included Petrimonas (9.3\%), Burkholderia (4.8\%), and
Stenotrophomonas (4.5\%). Similarly, the Azospirillum in the CC of CTC was more than in the OC of CTC. These results implied that antibiotic-resistant bacteria were more easily enriched under the MFC in closed-circuit. Petrimonas was described as a mesophilic, anaerobic, fermentative bacterium and observed in UASB reactors and it could produce acetate, hydrogen, and $\mathrm{CO}_{2} \cdot{ }^{45}$ In addition, Dokdonella was almost detected in all MFCs, and its abundance in the CC of OTC $(2.9 \%)$ or CTC $(4.1 \%)$ was more than that in OC of OTC $(1.7 \%)$ or CTC $(0.3 \%)$. This bacterium is electrochemically active such that it is responsible for the electron transfer via an electrode. ${ }^{46}$

OTC and CTC are very similar in structure, but the structural formula of CTC contained chlorine atoms, different group might be responsible for the difference in microbial communities of the anodic biofilm between OTC and CTC. The microorganisms of anodic biofilm in the CC can be divided into two functions groups: exoelectrogenic bacteria and antibiotics
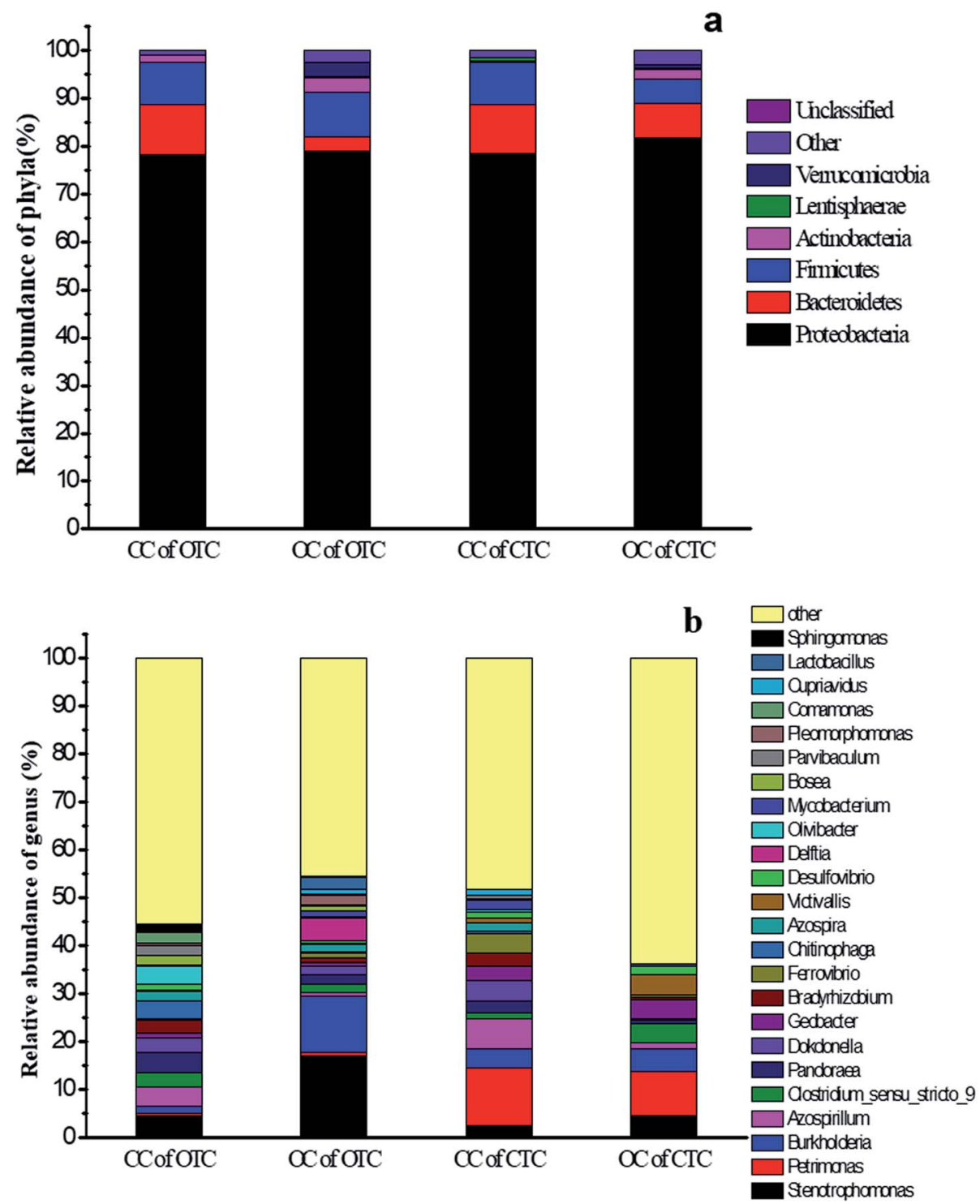

Fig. 9 (a) Relative abundance of bacterial phylum communities and (b) genus communities in the anode after MFC treatment by OTC and CTC in the $\mathrm{CC}$ and $\mathrm{OC}$ groups. 
degradation-related bacteria. In CC of CTC, exoelectrogenic bacteria (Dokdonella) and CTC-degradation-related bacteria (Petrimonas, Azospirillum and Stenotrophomonas) was synergistic effect on CTC degradation. In CC of OTC, exoelectrogenic bacteria was also Dokdonella and OTC-degradation-related bacteria was Azospirillum and Stenotrophomonas. The highest abundance of Petrimonas was existed in CTC. This is mainly due to glucose as a co-substrate to help the microbial acclimatization in the gradient acclimation stage, and Petrimonas was capable to degrade glucose and provide electron donor (lactate, acetate, and hydrogen) for dichlorination, ${ }^{47}$ and dechlorination was an important step in CTC degradation process, thus a large number of Petrimonas enrichment at the anode in MFC with CTC. The benzene ring of dechlorinated CTC or OTC was opened and broken down into small molecular organic acids by Azospirillum and Stenotrophomonas. These small molecular organic acids can be utilized by electrogenic bacteria for electricity generation and the oxidation rate of those was accelerated in CC group, thus the abundance of Dokdonella in CC group was higher than that in OC group. And the accelerated removal rates of small molecular organic acids could increase the metabolic reaction rates of antibiotics-degradation-related bacteria, thus the degradation of antibiotics was fastest in the MFC. In the OC group, electrons cannot be transferred quickly through external circuits, the electrons were transferred slowly to fix nitrogen and maintain anaerobic conditions by Burkholderia. Therefore, enriching antibiotic-resistant electrochemically active bacterium under the MFC in CC is advantageous to accelerate the removal of antibiotics.

\section{Conclusion}

CTC and OTC can be rapidly degraded in MFC under CC, and the removal efficiency of CTC and OTC reached $74.2 \%$ and $78 \%$ within 7 days. The degradation of CTC and OTC followed the first-order kinetic model within $12 \mathrm{~h}$. However, the removal rate of OTC was superior to that of CTC. Meanwhile, the possible degradation pathways for both antibiotics were analyzed. The toxicity of the degradation products of CTC and OTC was evaluated using the zebrafish. Based on the electronic transmission and biological domestication of MFC, the zebrafish assessment showed the nontoxicity of the degradation products. Compared with the traditional removal of antibiotics, the results proved that MFC was an efficient and environment-friendly method for removing CTC and OTC in wastewater.

\section{Conflicts of interest}

There are no conflicts to declare.

\section{Acknowledgements}

This work was supported by the Major projects of Natural Science Research in Jiangsu Province (Grant No. 15KJA530002); the Technology Supporting Program of Jiangsu Province (Grant No. BE2015167); Fund from the State Key Laboratory of Materials-Oriented Chemical Engineering (ZK201605) and the
Priority Academic Program from Development of Jiangsu Higher Education Institutions (XTD1816).

\section{References}

1 N. S. Lewis and D. G. Nocera, Proc. Natl. Acad. Sci. U. S. A., 2006, 103, 15729-15735.

2 Q. Q. Zhao, G. G. Ying, C. G. Pan, Y. S. Liu and J. L. Zhao, Environ. Sci. Technol., 2015, 49, 6772-6782.

3 S. Yahiat, F. Fourcade, S. Brosillon and A. Amrane, Int. Biodeterior. Biodegrad., 2011, 65, 997-1003.

4 M. Prateeksha, S. Sandip and P. Anjali, Chem. Eng. J., 2015, 276, 155-165.

5 H. C. Lützft, B. H. Sørensen and S. E. Jørgensen, Arch. Environ. Contam. Toxicol., 1999, 36, 1-6.

6 B. Halling-Sensen, Chemosphere, 2000, 40, 731-739.

7 X. Pan, Z. M. Qiang, W. W. Ben and M. X. Chen, Chemosphere, 2011, 84, 695-700.

8 L. J. Zhou, G. G. Ying and J. L. Zhao, Environ. Pollut., 2011, 159, 1877-1885.

9 C. Gu and K. G. Karthikeyan, Environ. Sci. Technol., 2005, 39, 2660-2667.

10 H. Lee, E. Lee and C. H. Lee, J. Ind. Eng. Chem., 2011, 17, 468473.

11 K. A. Loftin and C. D. Adams, J. Environ. Qual., 2008, 37, 378386.

12 L. Bin and Z. Tong, Environ. Sci. Technol., 2010, 44, 34683473.

13 R. Andreozzi and V. Caprio, Catal. Today, 1999, 53, 51-59.

14 J. Hollender, S. G. Zimmermann, S. Koepke and M. Krauss, Environ. Sci. Technol., 2009, 43, 7862-7869.

15 P. Nolwenn, O. Juan and A. Abdeltif, Process Biochem., 2009, 44, 1302-1306.

16 B. E. Logan and J. M. Regan, Trends Microbiol., 2006, 14, 512518.

17 J. K. Hyung, S. P. Hyung, S. H. Moon, I. S. Chang, M. Kim and H. K. Byung, Enzyme Microb. Technol., 2002, 30, 145-152.

18 H. J. Kim, M. S. Hyun, I. S. Chang and B. H. Kim, J. Microbiol. Biotechnol., 1999, 9, 365-367.

19 L. Hong and C. Shaoan, Environ. Sci. Technol., 2005, 39, 658662.

20 Y. J. Feng, X. Wang, B. E. Logan and H. Lee, Appl. Microbiol. Biotechnol., 2008, 78, 873-880.

21 T. S. Song, X. Y. Wu and C. C. Zhou, Bioprocess Biosyst. Eng., 2014, 37, 133-138.

22 Y. Luo, G. L. Liu, R. D. Zhang and C. P. Zhang, J. Power Sources, 2010, 195, 190-194.

23 S. Zhang, H. L. Song, X. L. Yang, K. Y. Yang and X. Y. Wang, Chemosphere, 2016, 164, 113-119.

24 J. Wang, M. F. He, D. L. Zhang, Z. Y. Ren, T. S. Song and J. J. Xie, $R S C A d v .$, 2017, 7, 44226-44233.

25 H. Zhang, Y. Y. Zhang and D. P. Li, Bioresour. Technol., 2017, 229, 104-110.

26 Y. Kong, B. Liang, H. Yun, H. Y. Cheng, J. C. Ma, M. H. Cui, A. J. Wang and N. Q. Ren, Water Res., 2015, 72, 281-292.

27 K. Li, J. Q. Wu, L. L. Jiang, L. Z. Shen, Z. Lv and M. F. He, Chemosphere, 2017, 171, 40-48. 
28 H. Y. Chen, Y. D. Liu and B. Dong, Bioprocess Biosyst. Eng., 2018, 41, 47-56.

29 M. J. Fan, N. N. Zhou, P. W. Li, L. L. Chen, Y. W. Chen and S. B. Shen, J. Hazard. Mater., 2017, 321, 791-800.

30 M. U. Ernest and C. A. Reddy, Microbial Degradation of Xenobiotics, 2011, vol. 10, pp. 31-66.

31 F. Bonfatti, S. Ferro, F. Lavezzo, M. Malacarne and G. Lodi, J. Electrochem. Soc., 2000, 147, 592-596.

32 C. J. Israelides, A. G. Vlyssides, V. N. Mourafetib and G. Karvounib, Bioresour. Technol., 1997, 61, 163-170.

33 C. B. Ding, Y. Zhao, J. P. Zhang, Z. G. Peng, D. Q. Song and J. D. Jiang, Int. J. Mol. Med., 2015, 35, 791-797.

34 C. Marina and S. Nikolaus, Mol. Cancer Res., 2010, 3, 517523.

35 A. F. Schier, Nature, 2013, 496, 443-444.

36 T. Søeborg, F. Ingerslev and S. B. Halling, Chemosphere, 2004, 57, 1515-1524.

37 H. S. Bent, L. Anne, I. Flemming, B. Paul and T. Jette, Chemosphere, 2003, 50, 1331-1342.

38 J. Diana, L. Vandenbosch, B. D. Spiegeleer, J. Hoogmartens and E. Adams, J. Pharm. Biomed. Anal., 2005, 39, 523-530.
39 B. Sørensen, G. Sengeløv and J. Tjørnelund, Arch. Environ. Contam. Toxicol., 2002, 42, 263-271.

40 G. Chen, L. Zhao and Y. H. Dong, J. Hazard. Mater., 2011, 193, 128-138.

41 E. Y. Jeon, H. L. Jung, M. Y. Kyung, C. J. Young, K. O. Deok and B. P. Jin, Process Biochem., 2012, 3, 941-947.

42 L. P. Huang, M. John and Q. Xie, Bioresour. Technol., 2011, 102, 316-323.

43 L. Lopez, L. S. Urzua, M. A. Mascarua, I. H. Camacho and J. C. Mellado, Soil Biol. Biochem., 1989, 21, 651-655.

44 Y. Bashan, H. Gina and E. Luz, Can. J. Microbiol., 2004, 50, 521-577.

45 E. Castelló, Y. S. C. García, T. Iglesias, G. Paolino, J. Wenzel, L. Borzacconi and C. Etchebehere, Int. J. Hydrogen Energy, 2009, 34, 5674-5682.

46 C. Xiang, Y. P. Quan and K. T. Quan, Chem. Eng. J., 2012, 210, 150-156.

47 Y. Zhang, M. Hu, P. F. Li, X. Wang and Q. J. Meng, Appl. Microbiol. Biotechnol., 2015, 99, 1977-1987. 